\title{
Editorial
}

\section{Computational Methods for Fracture}

\author{
Timon Rabczuk, ${ }^{1,2}$ Stéphane P. A. Bordas, ${ }^{3,4}$ and Goangseup $\mathrm{Zi}^{2}$ \\ ${ }^{1}$ Bauhaus Universität Weimar, 99423 Weimar, Germany \\ ${ }^{2}$ Korea University, Seoul 136-701, Republic of Korea \\ ${ }^{3}$ Faculty of Science, Technology and Communication, Research Unit in Engineering Science, University of Luxembourg, \\ Campus Kirchberg, G 007, 6 rue Richard Coudenhove-Kalergi, 1359 Luxembourg, Luxembourg \\ ${ }^{4}$ Cardiff School of Engineering, Cardiff University, Room W2.43, Queen's Buildings, The Parade, Cardiff 24 3AA, UK
}

Correspondence should be addressed to Timon Rabczuk; timon.rabczuk@uni-weimar.de

Received 15 May 2014; Accepted 15 May 2014; Published 20 July 2014

Copyright (C) 2014 Timon Rabczuk et al. This is an open access article distributed under the Creative Commons Attribution License, which permits unrestricted use, distribution, and reproduction in any medium, provided the original work is properly cited.

The numerical study of fracture has far-reaching applications throughout engineering and science. Its importance is becoming even more significant that engineers and materials scientists are thriving to devise new lighter and stronger materials from the bottom up.

Simulating fracture requires devising suitable models, discretizing the resulting partial differential equations, and solving them numerically. Each of those three steps poses its own difficulties which have been tackled in various ways, both academically and for practical applications.

This special issue deals with a range of such models: discretization and solution methods applied to a number of problems ranging from rock mechanics to surgical simulation requiring tackling various loading spectra, ranging from fast dynamics to quasistatic loading, and leading to a number of different failure modes, from brittle to ductile fracture.

The topics of this issue can be decomposed into five groups:

Models have been developed with special emphasis on rock mechanics and multi-field problems in fracture $[1,2]$ with applications, for example to coupled thermohydromechanical model of jointed hard rock for compressed air energy storage, and to rock failure [3].

Discretization methods have been heavily investigated, to address the difficulties faced by the standard finite element method [4], in particular, associated with remeshing as the cracks evolve. The issue discusses recent developments in meshless methods, including a posteriori error estimation and adaptive methods.
Dynamic fracture is in itself a wide field of study. Papers in this issue focus on explicit dynamics and tackle, in particular, the issue of energy dissipation during crack growth and the simulation of fracture in thin shells due to dynamic and implosive or explosive loading.

Model reduction methods have recently been developed for fracture simulations. Algebraic model reduction such as the proper orthogonal decomposition (POD) is not inherently well-suited to such problems [5-8]. This issue discusses one possibility relying on the combination of POD with meshfree methods.

Parameter identification in fracture mechanics is a topic of special interest in practical applications and a necessary step to provide convincing and predictive modeling and simulation tools. Two methods are discussed in this issue, both for crack parameter identification and multifield problems in fracture.

Through these five topics, we believe the issue gives a fair reflection of the current state of the art, with a heavy focus on modeling and simulation methods, although covering the whole spectrum of applications and methodologies would require a much more substantial volume. 


\section{References}

[1] X. Zhuang, C. Augarde, and S. Bordas, "Accurate fracture modelling using meshless methods, the visibility criterion and level sets: Formulation and 2D modelling," International Journal for Numerical Methods in Engineering, vol. 86, no. 2, pp. 249268, 2011.

[2] X. Zhuang, C. E. Augarde, and K. M. Mathisen, "Fracture modeling using meshless methods and levels sets in 3D: framework and modeling," International Journal for Numerical Methods in Engineering, vol. 92, no. 11, pp. 969-998, 2012.

[3] H. Zhu, X. Zhuang, Y. Cai, and G. Ma, "High rock slope stability analysis using the enriched meshless Shepard and least squares method," International Journal of Computational Methods, vol. 8, no. 2, pp. 209-228, 2011.

[4] Y. Cai, X. Zhuang, and C. Augarde, "A new partition of unity finite element free from the linear dependence problem and possessing the delta property," Computer Methods in Applied Mechanics and Engineering, vol. 199, no. 17-20, pp. 1036-1043, 2010.

[5] P. Kerfriden, P. Gosselet, S. Adhikari, and S. P. A. Bordas, "Bridging proper orthogonal decomposition methods and augmented Newton-Krylov algorithms: an adaptive model order reduction for highly nonlinear mechanical problems," Computer Methods in Applied Mechanics and Engineering, vol. 200, no. 5-8, pp. 850-866, 2011.

[6] P. Kerfriden, J. C. Passieux, and S. P. A. Bordas, "Local/global model order reduction strategy for the simulation of quasibrittle fracture," International Journal for Numerical Methods in Engineering, vol. 89, no. 2, pp. 154-179, 2012.

[7] P. Kerfriden, O. Goury, T. Rabczuk, and S. P. A. Bordas, "A partitioned model order reduction approach to rationalise computational expenses in nonlinear fracture mechanics," Computer Methods in Applied Mechanics and Engineering, vol. 256, pp. 169-188, 2013.

[8] P. Kerfriden, K. M. Schmidt, T. Rabczuk, and S. P. A. Bordas, "Statistical extraction of process zones and representative subspaces in fracture of random composites," International Journal for Multiscale Computational Engineering, vol. 11, no. 3, pp. 253287, 2013. 


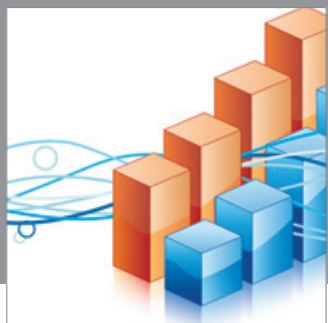

Advances in

Operations Research

mansans

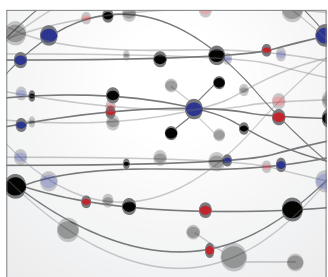

The Scientific World Journal
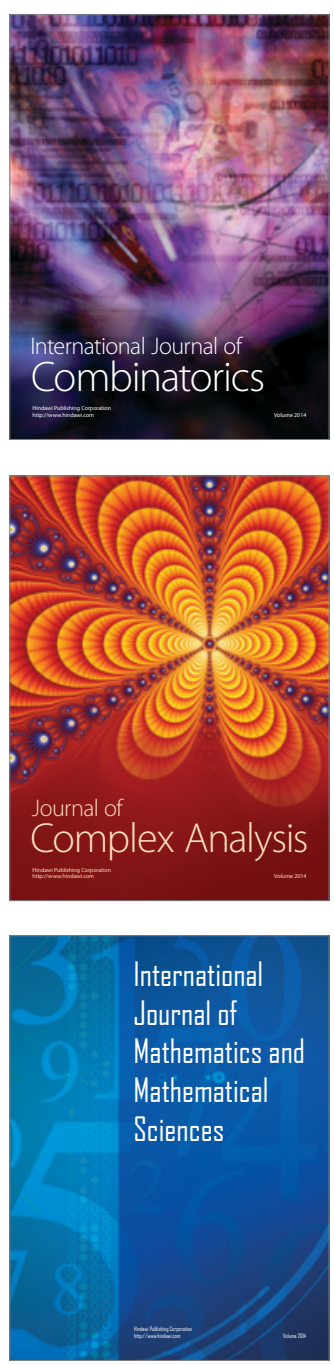
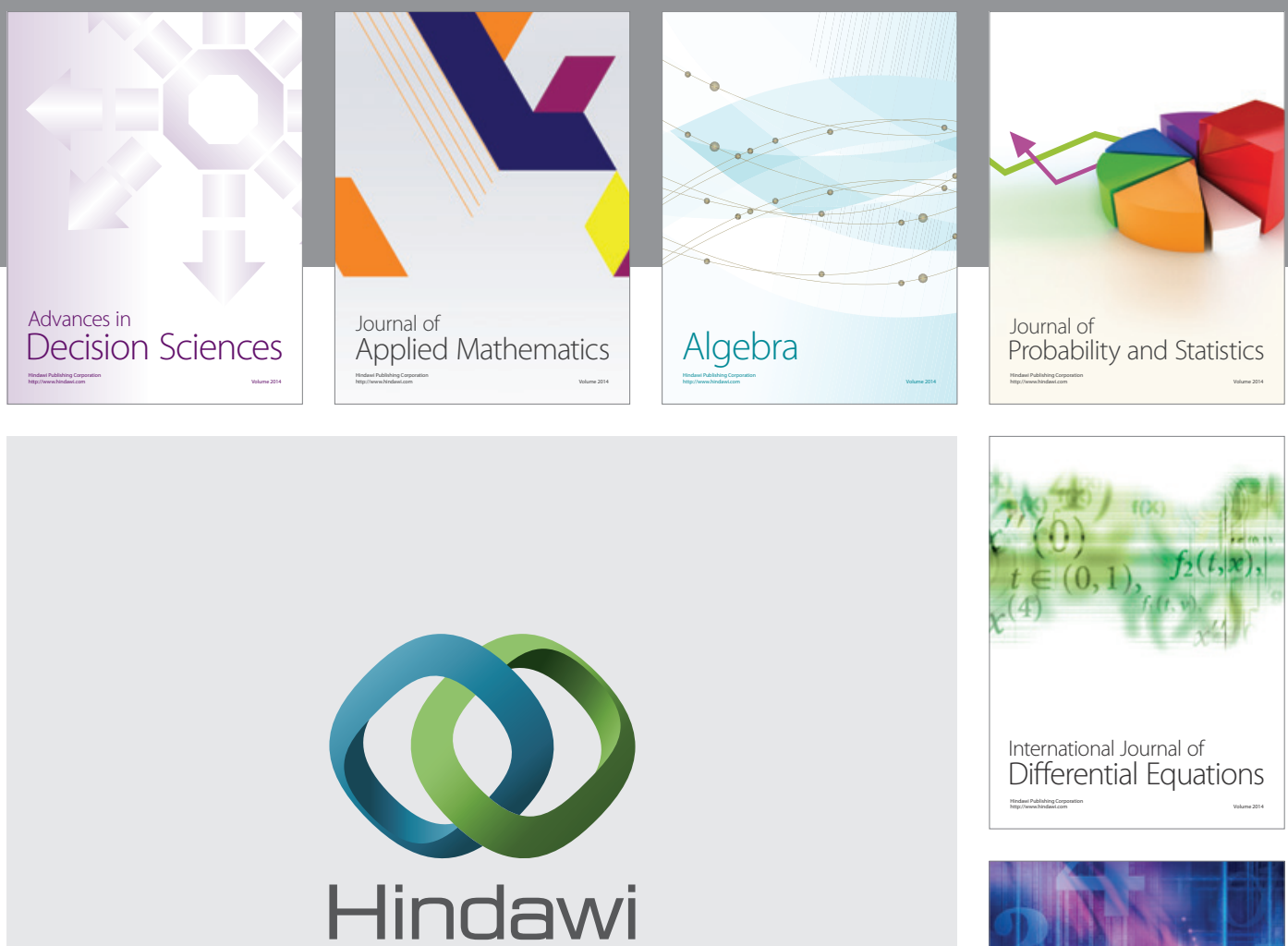

Submit your manuscripts at http://www.hindawi.com
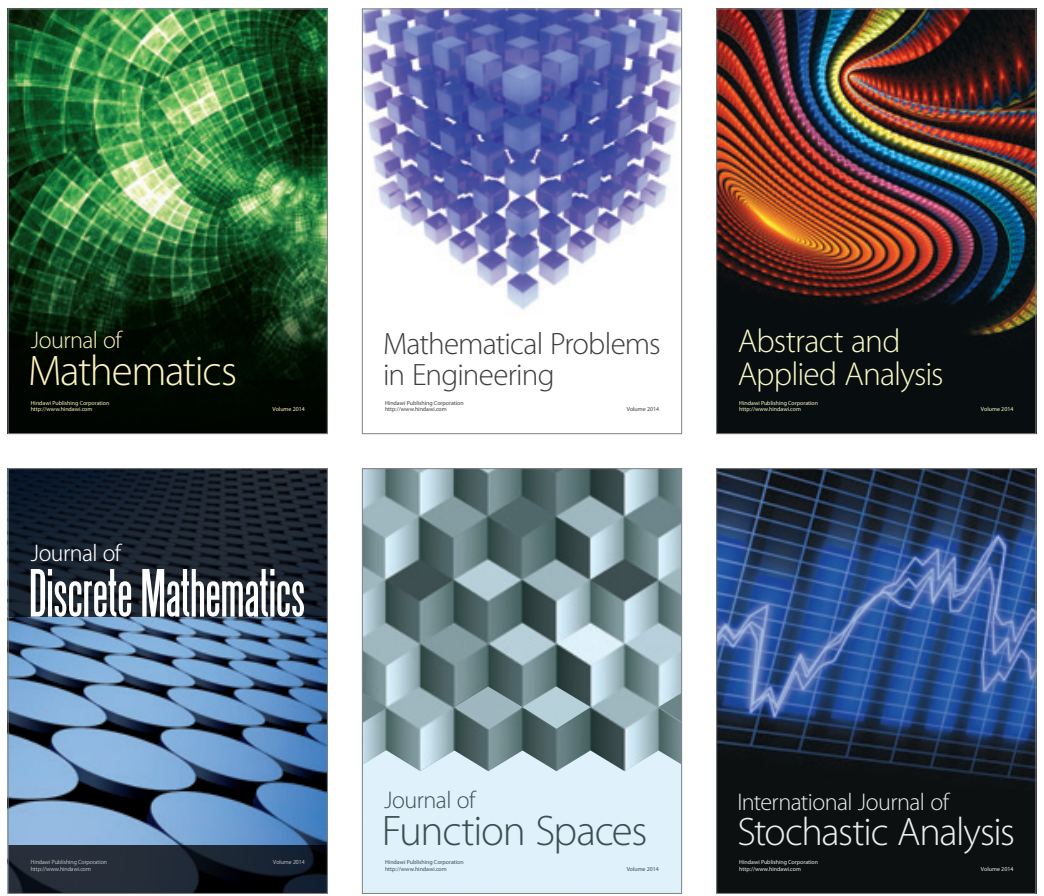

Journal of

Function Spaces

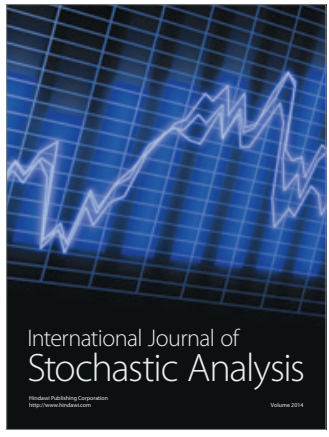

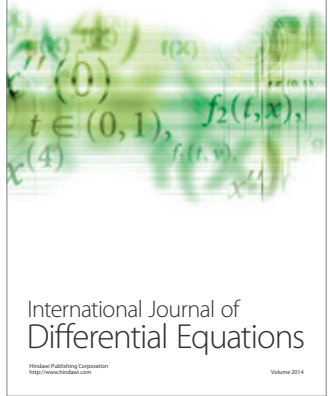
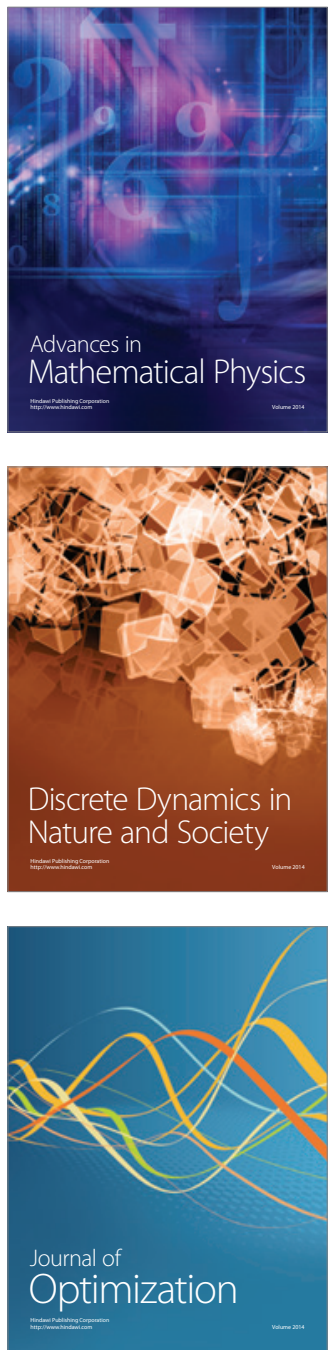